\title{
MRI characteristics of proctitis in Crohn's disease on perianal MRI
}

\author{
Charlotte J. Tutein Nolthenius, ${ }^{1,3}$ Shandra Bipat, ${ }^{1}$ Banafsche Mearadji, ${ }^{1}$ \\ Anje M. Spijkerboer, ${ }^{1}$ Cyriel Y. Ponsioen, ${ }^{2}$ Alexander D. Montauban van Swijndregt, ${ }^{3}$ \\ Jaap Stoker ${ }^{1}$
}

\author{
${ }^{1}$ Department of Radiology, Academic Medical Center, University of Amsterdam, PO Box 22660, 1100 DD Amsterdam, \\ The Netherlands \\ ${ }^{2}$ Department of Gastroenterology and Hepatology, Academic Medical Center, University of Amsterdam, PO Box 22700, \\ 1100 DE Amsterdam, The Netherlands \\ ${ }^{3}$ Department of Radiology, OLVG, PO Box 99550, 1090 HM Amsterdam, The Netherlands
}

\begin{abstract}
Purpose: Multiple features have been described for assessing inflammation in Crohn's disease (CD) in MR enterography, but have not been validated in perianal magnetic resonance imaging (MRI). Retrospectively, we studied which MRI features are valuable in assessing proctitis.

Materials and methods: $\mathrm{CD}$ patients ( $\geq 18$ years) who underwent colonoscopy (reference standard) and perianal fistula MRI within 8 weeks were included. Seventeen MRI features were blindly scored by three observers and correlated to endoscopy (regression analysis). Reproducibility (multirater kappa, intraclass correlation coefficient) was determined for all three observer pairs. MRI features were considered relevant when significantly correlated to endoscopy for $\geq 2$ observers, and reproducibility was $\geq 0.40$ for $\geq 2$ observer pairs.

Results: Perianal MRI of $58 \mathrm{CD}$ patients were included. Wall thickness, rectal mural fat, creeping fat, and size of mesorectal lymph nodes showed a significant correlation with endoscopy for $\geq 2$ observers $(p=0.000-0.023$, $p=0.011-0.172, \quad p=0.007-0.011$ and $p=0.000$ 0.005 , respectively) with a kappa/intraclass correlation coefficient of $\geq 0.60$ for $\geq 2$ observer pairs. Perimural T2 signal and perimural enhancement significantly correlated to endoscopy (all $p$ values $\leq 0.05$ ) for all three observers and the reproducibility was $\geq 0.40$ for $\geq 2$ observer pairs. Mural T2 signal and degree and pattern of $\mathrm{T} 1$ enhancement showed significant correlation to
\end{abstract}

Correspondence to: Charlotte J. Tutein Nolthenius; email: c.j.tutein nolthenius@amc.uva.nl endoscopy for two observers, but with poor to moderate reproducibility.

Conclusion: Wall thickness, mural fat, and mesorectal features (perimural T2 signal, perimural enhancement, creeping fat, and size of mesorectal lymph nodes) had significant correlation to endoscopy and were reproducible in diagnosing proctitis. Some established luminal features in MRE were considered not useful.

Key words: Proctitis - Crohn disease - Magnetic resonance imaging - Rectum-Inflammatory bowel disease

Magnetic resonance imaging (MRI) of the perianal region has proven to be a valuable tool in diagnosing perianal fistulas in patients with Crohn's disease, with accuracies reported up to $93 \%$ in classifying fistulas and $96 \%$ in delineating abscesses [1, 2]. The anatomy and complexity of the fistula tract can precisely be depicted which is important for treatment planning [3]. Preoperative MRI has shown to reveal additional and clinically relevant information, thereby reducing recurrence rates after fistula surgery [1, 4]. Another important issue in treatment planning is the concomitant presence of proctitis. Proctitis is defined as an inflammation of the rectum, approximately $12-15 \mathrm{~cm}$ from the dentate line. In the presence of proctitis, the chance of fistula healing is reduced, and therefore, a more aggressive medical therapy should be started and surgery should be avoided $[3,5,6]$.

Extensive research revealed multiple MRI features and scoring systems able to accurately assess inflammation in luminal Crohn's disease [7-9]. These features have 
been assessed on MR enterography or MR colonography, and have not been tested in dedicated pelvic MRI, which is limited by a different scan protocol (small FOV, other sequences) and the absence of luminal contrast. As many patients with perianal fistulas will undergo a pelvic MRI before start of treatment, diagnosing the presence and degree of proctitis on this MRI could be of additional value $[2,3]$.

In our retrospective study, we aimed to identify the MRI features of proctitis on a dedicated pelvic MRI, and to determine the reproducibility of the different MRI features.

\section{Materials and methods}

\section{Patients}

From January 2001 until February 2014, we searched the endoscopy database (EndoAlpha Documentation, Olympus Nederland BV, Zoeterwoude, The Netherlands) of the Academic Medical Center, Amsterdam, The Netherlands, for patients ( $\geq 18$ years of age) with known Crohn's disease who underwent a proctoscopy, sigmoidoscopy, or colonoscopy and who also underwent a dedicated pelvic MRI according to our standard MRI perianal fistula protocol within either 8 weeks prior to or after endoscopy. We chose this eight-week interval balancing inclusion versus a satisfactory interval. Patients were included if the endoscopy report mentioned the rectum, either with regard to the diagnosis of proctitis, rectal inflammation, or rectitis or with regard to no signs of rectal inflammation at all. Patients could only be included once. In that case, the most recent MRI was chosen. For consistency, MRIs performed with an endocoil or with an incomplete scan protocol were excluded. Electronic medical records were searched by a research fellow (CTN) and relevant information was noted (time of diagnosis, use of medication during the examinations, previous surgery). Patients with change in therapy, either medical or surgical, in the period between endoscopy and MRI were excluded. All included MRI scans were blinded and randomly ordered.

The requirement for review by the Medical Ethical Committee or informed consent was waived because of the retrospective nature of this study with pre-existing data.

\section{Reference standard}

With no access to clinical information or MRI scans, we evaluated the endoscopy reports of all included patients and performed a classification of lesion severity by considering three categories: grade (1) absence of lesions; grade (2) presence of inflammatory lesions without ulceration, including erythema, oedema, pseudopolyps, and aphthae; and grade (3) presence of superficial or deep ulcerations [10]. The presence or absence of fistulas and anal stenosis was also noted. Uncertainties were resolved by the expert opinion of a gastroenterologist (CY; 20 years of experience), with inflammatory bowel disease as subspecialty, with access to all endoscopical information, including endoscopy images.

\section{MRI protocol}

All MRIs were performed at 1.5T (Signa Horizon Echospeed, LX 9.0, General Electric Medical Systems, Milwaukee, WI, USA and MAGNETOM Avanto, Siemens Healthcare, Erlangen, Germany) and at 3T (INTERA, Philips Medical Systems, Best, the Netherlands) without bowel preparation, except 4-h fasting. Patients were scanned in supine position using a torso phased-array surface coil. Sagittal, coronal, and transversal sequences were performed with the coronal and transversal sequences angulated parallel and perpendicular to the anal canal, respectively. The scan protocol consisted of T2-weighted Turbo Spin-Echo sequences in the sagittal, coronal, and transversal planes, a fat-suppressed transversal T2-weighted TSE sequence and a fat-suppressed transversal T1-weighted TSE sequence after intravenous gadolinium. For a detailed description of all MRI parameters see Appendix A.

\section{Observers}

All MRI scans were blinded and retrospectively evaluated by three abdominal radiologists with different relevant experience levels: Observer 1 (BM; abdominal radiologist for 9 years including approximately 500 perianal fistula MRIs and $500 \mathrm{MR}$ enterographies) years, observer 2 (AS; radiologist for 20 years including approximately 300 perianal fistula MRIs and $300 \mathrm{MR}$ enterographies), and observer 3 (JS; abdominal radiologist for 21 years including approximately 1300 perianal fistula MRIs and $800 \mathrm{MR}$ enterographies). No clinical or endoscopy findings were provided, except for the presence or absence of perianal fistulas. . Before start, the observers read a document explaining the different MRI features to be evaluated with examples obtained from the previous literature and cases from a different dataset followed by a joined session discussing the features led by a fellow researcher (CTN) and the most experienced abdominal radiologist (observer 3: JS) (Fig. 1) [7, 11-14]. Five example cases from a different dataset were discussed. For evaluation of all cases, a digital questionnaire was developed (proctitis.co.nr).

\section{MRI features}

Quality of the scan and rectal distention were evaluated by the most experienced observer as poor (non-diagnostic), adequate (artifacts, but sufficient diagnostic quality), and good (no artifacts); and none (completely 

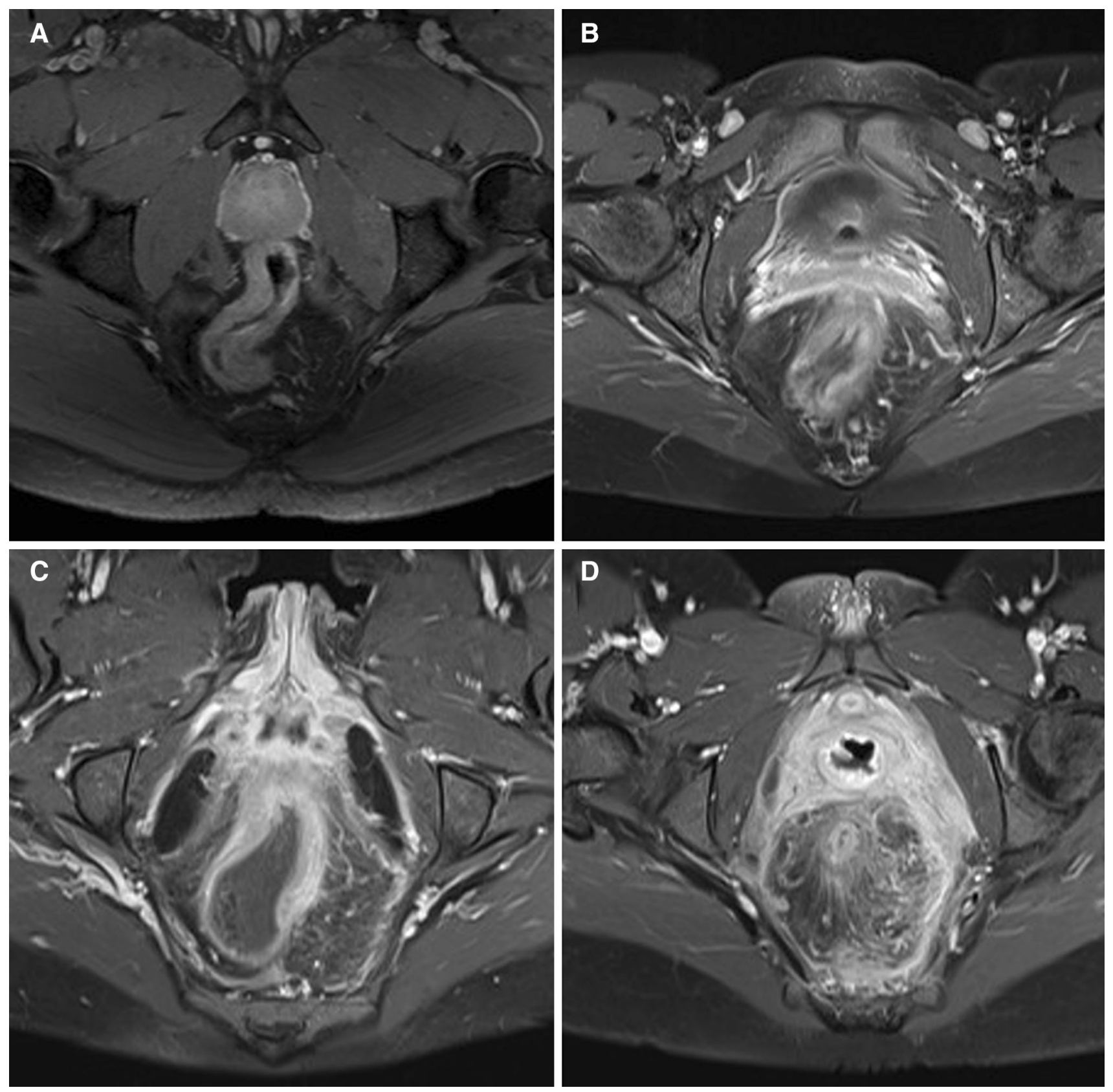

Fig. 1. Axial oblique fat-saturated post-contrast T1-weighted images of four different patients with Crohn's disease with different degrees of perimural enhancement. A Equivalent to normal fat tissue. B Minor enhancement. There is blurred demarcation of the bowel wall with or without mild increase of

collapsed rectum), moderate (some distension but no convex contours of the rectal wall), and good (convex contours of the rectal wall) assessed in the least distended part of the rectum, respectively. Seventeen MRI features (Table 1) were evaluated by all three readers. Features were selected according to MRI features described in the literature and those used in two published scoring systems on MRI in luminal Crohn's disease [7, 10, 11].

perimural fat tissue signal. C Moderate enhancement. Increase of perimural fat tissue signal but less than nearby vascular structures. D Marked enhancement. Perimural fat tissue signal approaches that of nearby vascular structures. Mesorectal fascia enhancement can be noted.

Definitions of certain features were adapted to be applicable in perianal MRI (Table 1). Additional items according to expert opinion were added: enhancement of perimural fat tissue (see Table 1; Fig. 1 for definition), creeping fat was defined as an increased amount of perirectal fat tissue and the comb sign as increased vascular structures in the perirectal fat, both scored on the sagittal images. The most affected part of the rectum was 


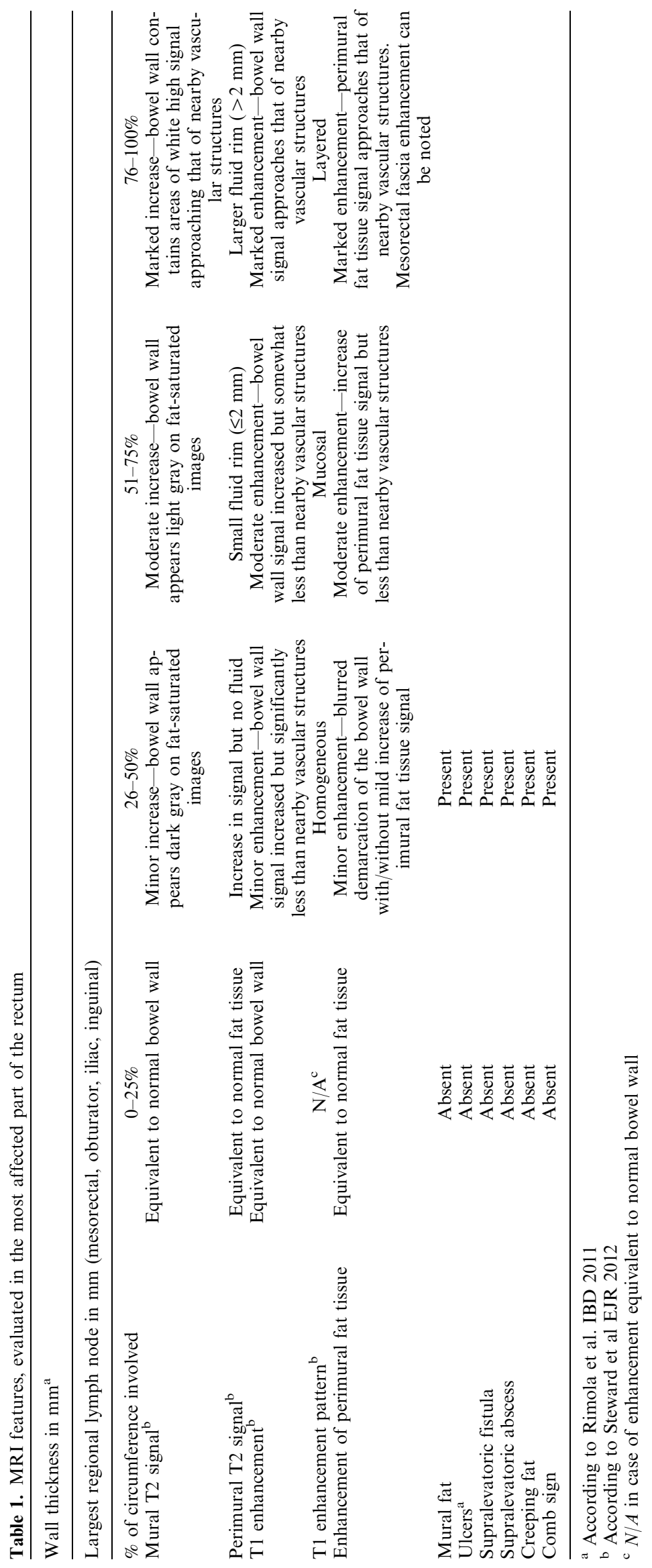


evaluated. Shortest axis of the largest lymph node per station was measured.

\section{Statistical analysis}

The maximum number of eligible patients in the given time period were included, and no sample size calculations were therefore performed. Extension of this time period to earlier period was not desirable, as the MRI protocol was different before 2001 (use of endocoil) and thereby not reflecting the practice nowadays.

Normality of continuous data was tested by visual assessment of the data. Normally distributed data were presented with means and SD. For non-normally distributed data, medians with interquartile ranges (IQR) were given.

\section{Interobserver agreement}

Several multirater analyses were performed for all features individually. To test the level of interobserver agreement for the separate MRI features between the three different pairs of radiologists, the appropriate measure was used. For all ordinal data, a weighted kappa coefficient was calculated per two raters. For the binominal data, a kappa coefficient was used calculated per two raters. For continuous data, an intraclass correlation coefficient was determined per two raters. Both kappa and intraclass correlation coefficient values were interpreted as follows: $0-0.20$, poor; $0.20-0.40$, fair; 0.40 0.60 , moderate; $0.60-0.80$, good; $0.80-1.00$, very good [15].

\section{Comparison of observers with reference standard}

Endoscopical reference standard was dichotomized in the absence of lesions (grade 0 ) versus proctitis (grades 1 and 2) because of limited size of study population. Associations were tested using regression analyses for ordinal or binominal MRI parameters. Comparison of continuous MRI parameters and endoscopical reference standard was performed using the Mann-Whitney $U$ test, as data were not normally distributed.

\section{Relevant MRI features}

MRI features with a significant correlation ( $p$ value of $\leq 0.05$ ) between the reference standard and at least two of three observers, and with a (weighted) kappa/intraclass correlation coefficient value of $\geq 0.60$ for at least two of three observer pairs, were identified and considered potentially relevant in diagnosing proctitis. In post hoc analysis, threshold for the kappa/intraclass correlation in considering a relevant feature was changed to $\geq 0.40$, because this concerns an initial study aimed at identifying potential relevant features and therefore sufficient features should be identified to be used in a future validation study.

All statistical analyses were performed with IBM SPSS Statistics version 20.0 for Windows (SPSS, Chicago, IL, USA) and Vassarstats.com (Richard Lowry, Poughkeepsie, NY, USA).

\section{Results}

\section{Patient and MRI characteristics}

Between January 2001 and February 2014, 106 Crohn's disease patients were extracted from the database who underwent perianal MRI within 8 weeks of endoscopy (Fig. 2). After exclusion, a total of 58 patients remained (Fig. 2), of which 21 (36\%) are male with a mean age of 38.7 (SD 12.6) at the time of MRI. The median time between MRI and endoscopy was 12 days ([IQR 6-21]; range $0-44)$. Thirty-two (55\%) had no signs of proctitis at endoscopy and $26(45 \%)$ had signs of proctitis, of which $19(33 \%)$ had non-ulcerative proctitis and 7 (12\%) ulcerative proctitis. Table 2 summarized the demographic and clinical data of patients included in the study.

Quality of the MRI scans was considered good in $74.1 \%(43 / 58)$ and adequate in $25.9 \%(15 / 58)$. There was no rectal distention in $43.1 \%(25 / 58)$, moderate distention in $34.5 \%(20 / 58)$, and good distention in $22.4 \%(13 /$ 58).

\section{Interobserver agreement}

Agreement between the three observer pairs is presented in Table 3. For size of mesorectal lymph nodes, the agreement for all three pairs ranged between good and very good (0.78 and 0.83$)$. Wall thickness, mural fat, and creeping fat showed good agreement for two of three observer pairs $(0.70-0.58-0.69,0.67-0.57-0.64$, and 0.48-0.69-0.76, respectively). Perimural T2 signal, supralevatoric extension of fistula, and abscess showed at least moderate agreement $(\geq 0.40)$ for all the three observer pairs. Perimural enhancement and size of inguinal lymph nodes showed at least moderate agreement for two of three observer pairs $(0.46-0.34-0.59$ and $0.65-$ $0.38-0.43)$.

\section{Comparison of observers with reference standard}

In Table 4, the comparison of continuous variables (upper part) and ordinal variables (lower part) with the reference standard are presented. Wall thickness was significantly smaller for all three observers in patients without proctitis than in patients with proctitis (observer 1: 6.0 vs. $9.0 \mathrm{~mm}, p=0.000$; observer $2: 8.0$ vs. $11.0 \mathrm{~mm}, p=0.023$; observer $3: 4.0$ vs. $10.0 \mathrm{~mm}$, $p=0.000)$. 


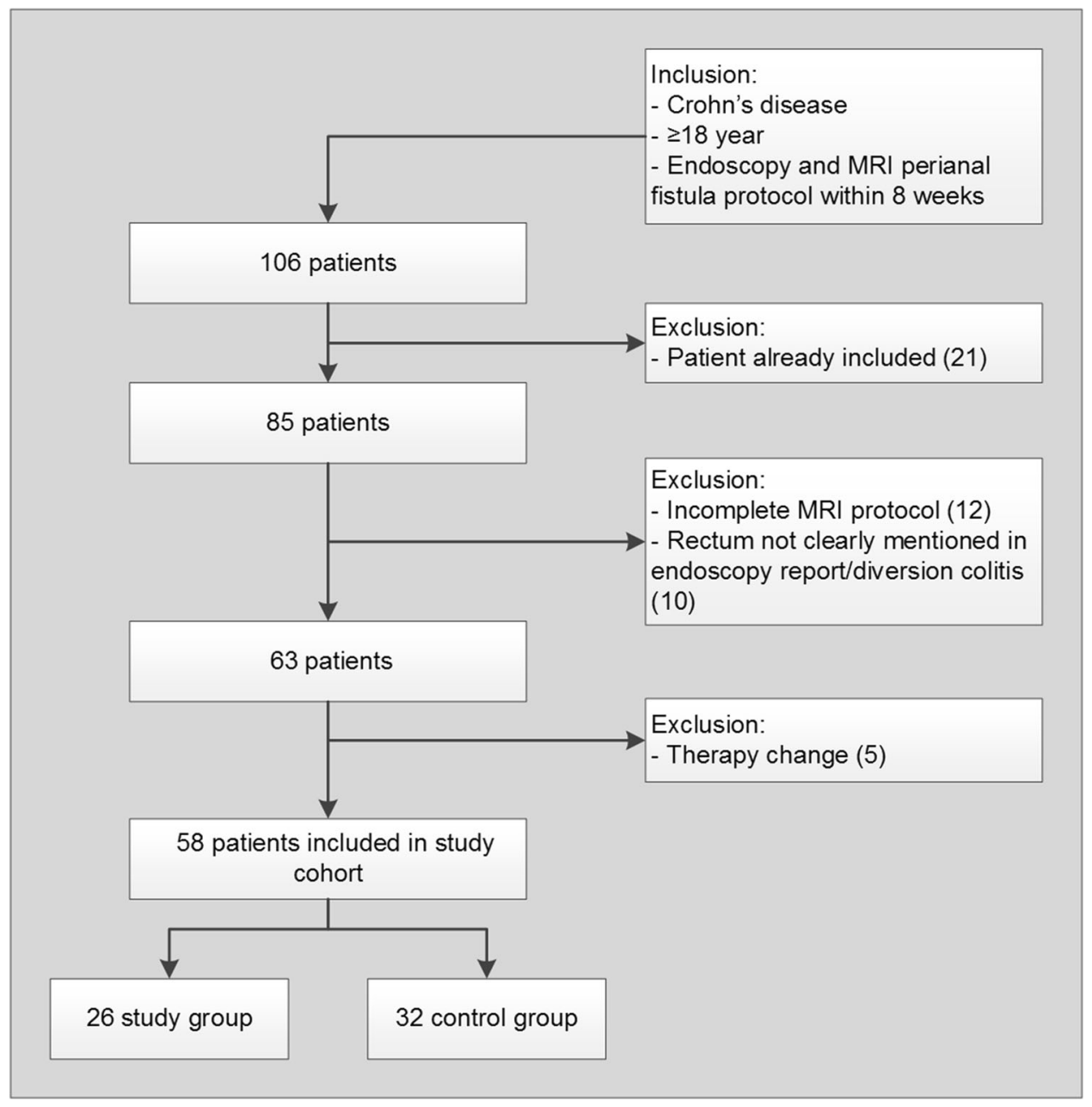

Fig. 2. Flow chart of search in hospitals' patient database.

Table 2. Demographic characteristics of the study population

\begin{tabular}{lll}
\hline & Study group & Control group \\
\hline No. (\%) of patients & $26(55)$ & $32(45)$ \\
Men (\%) & $13(50)$ & $8(25)$ \\
Women (\%) & $13(50)$ & $24(75)$ \\
Age at time of imaging (y), median (IQR) & $40(27-51)$ & $37(28-47)$ \\
Disease duration (y), median (IQR) & $7(4-11)$ & $7(3-21)$ \\
Days between endoscopy and MRI, median (IQR) & $13(7-23)$ & $11(4-20)$ \\
Previous surgery, no. (\%) of patients & $16(62)$ & $22(69)$ \\
Maintenance therapy, no. (\%) of patients & $18(69)$ & $23(72)$ \\
Antitumor necrosis factor, no. (\%) of patients & $5(19)$ & $11(34)$ \\
Steroids, no. (\%) of patients & $8(31)$ & $3(9)$ \\
Immunosuppressant, no. (\%) of patients & $12(46)$ & $12(38)$ \\
5-Aminosalicylic acid medications, no. (\%) of patients & $1(4)$ & $7(22)$ \\
Vedolizumab, no. (\%) of patients & $1(4)$ & 0 \\
Presence of fistula (on MRI) & $3(12)$ & $8(25)$ \\
None, no. (\%) of patients & $11(42)$ & $16(50)$ \\
Simple, no. (\%) of patients & $12(46)$ & $8(25)$ \\
Complex, no. $\%$ of patients & & $32(100)$ \\
Endoscopy diagnosis & 0 & 0 \\
Absence of lesions, no. (\%) of patients & $7(27)$ & 0 \\
Non-ulcerative lesions, no. (\%) of patients & $19(73)$ & \\
Ulcerations, no. (\%) of patients & &
\end{tabular}

$I Q R$ interquartile range 
Table 3. Multirater Kappa and intraclass correlation coefficient values between the observer pairs

\begin{tabular}{lccc}
\hline MRI features & 1 vs. 2 & 2 vs. 3 & 1 vs. 3 \\
\hline Wall thickness & $0.70(0.54-0.81)$ & $0.58(0.38-0.73)$ & $0.69(0.53-0.81)$ \\
Mesorectal lymph nodes & $0.83(0.72-0.89)$ & $0.78(0.66-0.87)$ & $0.83(0.72-0.89)$ \\
Obturator lymph nodes & $0.31(0.06-0.53)$ & $0.26(0.00-0.48)$ & $0.27(0.01-0.49)$ \\
Iliac lymph nodes & $0.38(0.14-0.58)$ & $0.38(0.14-0.58)$ & $0.24(-0.01-0.47)$ \\
Inguinal lymph nodes & $0.65(0.48-0.78)$ & $0.17(0.06-0.27)$ & $0.43(0.20-0.62)$ \\
\% of circumference involved & $0.16(0.03-0.29)$ & $43 \%(25 / 58)^{\mathrm{a}}$ & $0.47(0.27-0.67)$ \\
Mural T2 signal & $26 \%(15 / 58)^{\mathrm{a}}$ & $0.57(0.41-0.73)$ & $41 \%(24 / 58)^{\mathrm{a}}$ \\
Perimural T2 signal & $0.50(0.34-0.67)$ & $0.14(0.01-0.27)$ & $0.71(0.58-0.85)$ \\
T1 enhancement & $0.13(0.03-0.24)$ & $0.25(0.12-0.38)$ & $0.39(0.22-0.56)$ \\
T1 enhancement pattern & $0.13(0.02-0.25)$ & $0.34(0.17-0.50)$ & $0.43(0.25-0.61)$ \\
Perimural enhancement & $0.46(0.29-0.64)$ & $0.57(0.34-0.81)$ & $0.59(0.42-0.76)$ \\
Mural fat & $0.67(0.44-0.90)$ & $0.13(0-0.39)$ & $0.64(0.37-0.90)$ \\
Ulcers & $64 \%(37 / 58)^{\mathrm{a}}$ & $0.57(0.31-0.82)$ & $0.25(0-0.57)$ \\
Supralevatoric fistula & $0.48(0.24-0.72)$ & $0.53(0.24-0.82)$ & $0.59(0.39-0.79)$ \\
Supralevatoric abscess & $0.53(0.21-0.86)$ & $0.69(0.46-0.92)$ & 1 \\
Creeping fat & $0.48(0.18-0.77)$ & $0.20(0.03-0.36)$ & $0.76(0.53-0.98)$ \\
Comb sign & $0.18(0.01-0.34)$ & $0.55(0.32-0.78)$ \\
\hline
\end{tabular}

${ }^{a}$ Proportion of agreement calculated instead of kappa, because observed concordance is smaller than mean-chance concordance

Table 4. Comparison of observers with reference standard

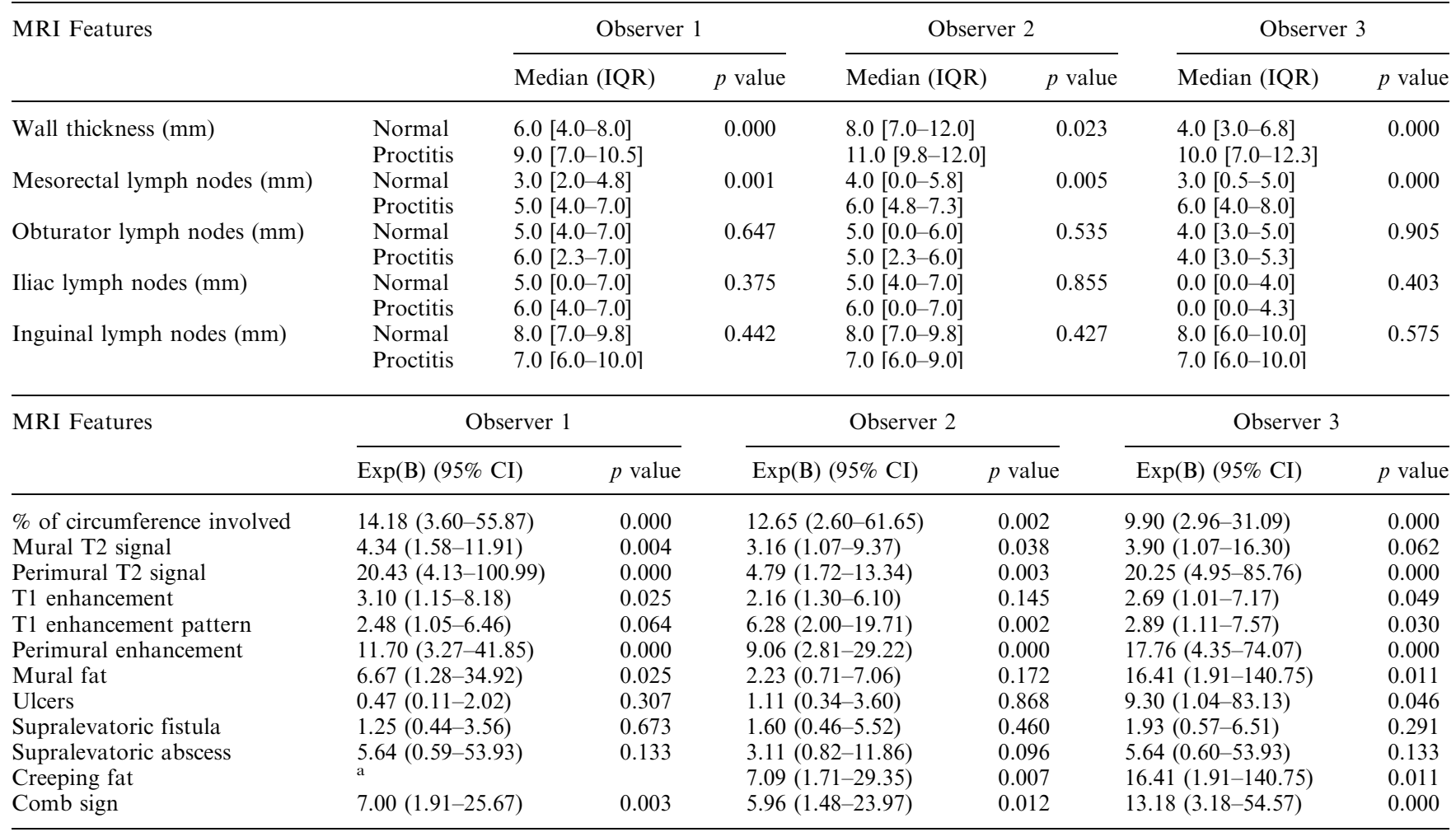

${ }^{a}$ Not calculated because of the presence of a zero in the crosstab

Mesorectal lymph nodes were smaller for all three observers in patients without proctitis than with proctitis (observer 1: 3.0 vs. $5.0 \mathrm{~mm}, p=0.001$; observer 2: 4.0 vs. $6.0 \mathrm{~mm}, p=0.005$; observer 3 : 3.0 vs. $6.0 \mathrm{~mm}$, $p=0.000)$.
Percentage of circumference involved, perimural T2 signal, perimural enhancement, and the presence of the comb sign showed also a significant correlation between all three observers and the endoscopy reference standard (Table 4). 


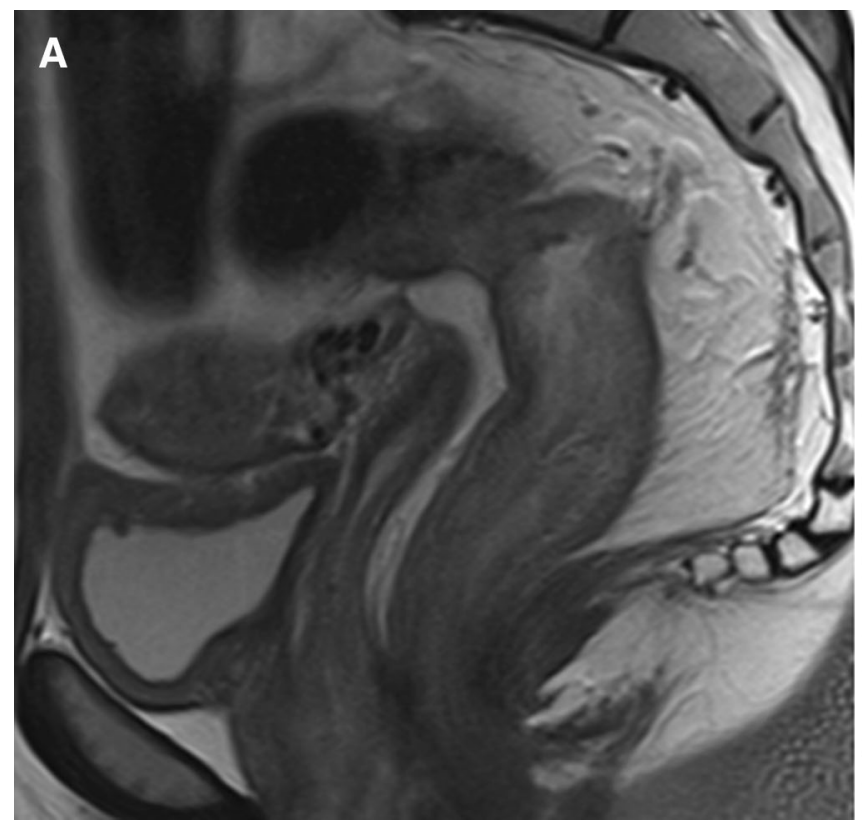

Fig. 3. Sagittal T2-weighted image of two different patients with Crohn's disease. A A 25-year-old female with ulcerative proctitis at endoscopy. The image shows increased amount of mesorectal fat tissue (creeping fat) and a subtle increase of

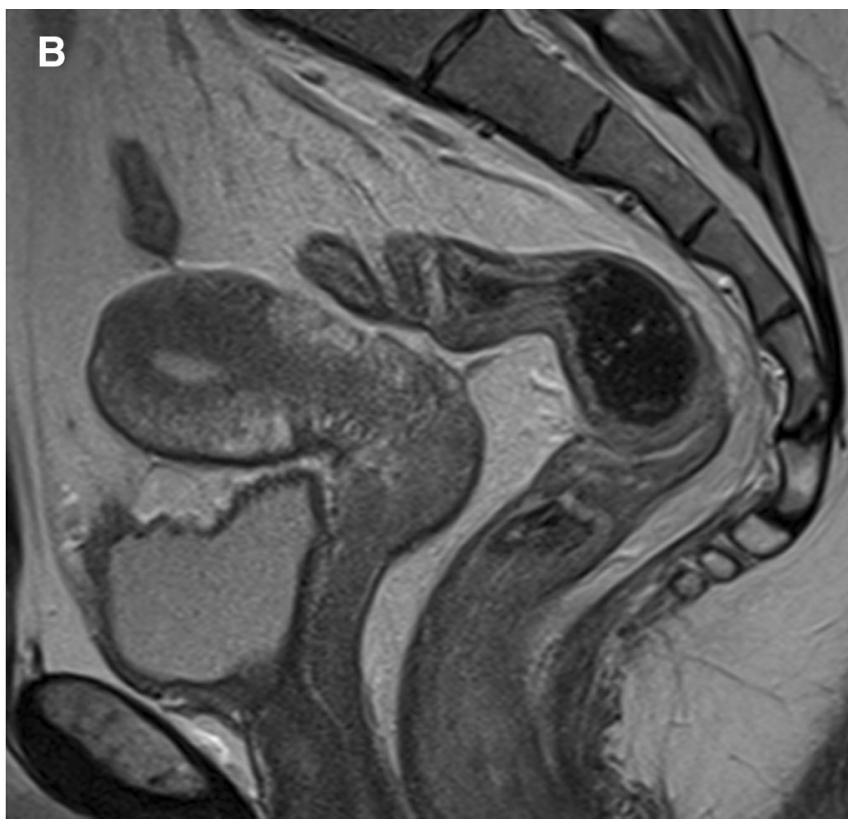

perimural vascularity ('comb sign') in addition to rectal wall thickening. B A 24-year-old female with no signs of proctitis at endoscopy. There is no increased amount of mesorectal fat tissue and the rectum shows no abnormal MRI features.
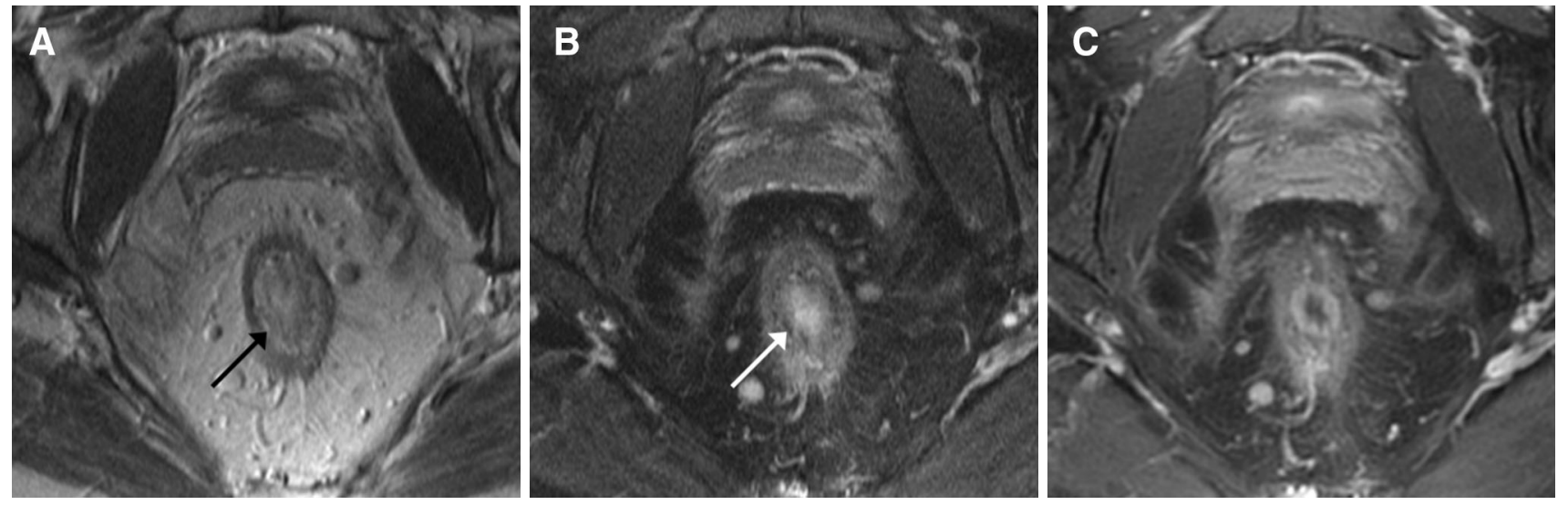

Fig. 4. A 53-year-old female with Crohn's disease and ulcerative proctitis at endoscopy. A Axial oblique T2-weighted image shows high mural signal intensity and B low signal intensity on axial oblique fat-saturated T2-weighted image

Mural T2 signal, T1 enhancement, T1 enhancement pattern, and creeping fat showed a significant correlation for two of three observers. Ulcers and supralevatoric extension of fistula and abscess did not show a significant correlation to the reference standard for all three observers. corresponding to mural fat (arrow). C Axial oblique fat-saturated post-contrast T1-weighted images shows moderate enhancement of the rectal wall and perimural fat tissue. In addition, wall thickening and multiple mesorectal lymph nodes are present.

\section{Relevant MRI features}

Based on predefined criteria, the following MRI features were considered most relevant in diagnosing proctitis (Tables 3, 4): wall thickness, size of mesorectal lymph nodes, mural fat, and creeping fat showed a significant 

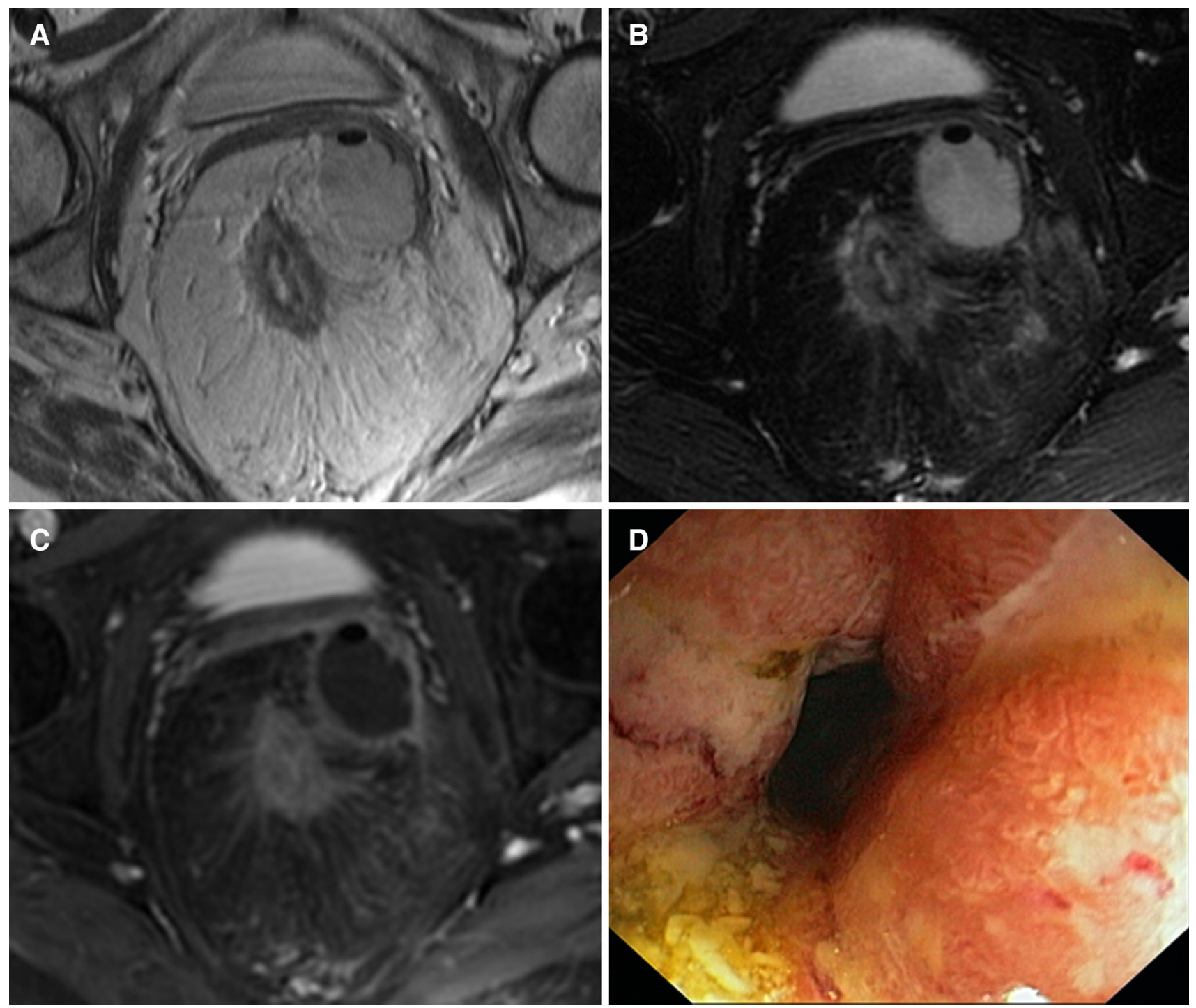

Fig. 5. A 49-year-old female with Crohn's disease. A Axial oblique T2-weighted image and $\mathbf{B}$ axial oblique fat-saturated T2-weighted image show rectal wall thickening, a marked increase of T2 signal intensity and a perimural large fluid rim $(>2 \mathrm{~mm})$. C Axial oblique fat-saturated post-contrast T1-

correlation between at least two of three observers and the endoscopy reference standard, as well as a kappa/ intraclass correlation coefficient of $\geq 0.60$ for at least two of three observer pairs (Figs. 3, 4). In post hoc analysis, a kappa/intraclass coefficient threshold of $\geq 0.40$ was considered, which included perimural T2 signal and perimural enhancement as they showed a moderate interobserver agreement for two of three observer pairs and a significant correlation with the reference standard for all three observers (Appendix B) (Fig. 5).

All other features (all other lymph nodes, \% of circumference involved, T1 enhancement (pattern), ulcers, supralevatoric fistula, and abscess and comb sign) did

weighted images obtained at the same level shows a moderate enhancement of the rectal wall and the perimural fat tissue. In addition, there is creeping fat and a supralevatoric abscess left anterolateral of the rectum on all three images. D Endoscopy showed ulcerative proctitis.

not fulfill our predefined criteria for relevancy. Although the correlation with endoscopy was significant for two observers, mural T2 signal and T1 enhancement (pattern) showed poor to moderate agreement.

\section{Discussion}

MRI features rectal wall thickness, mesorectal lymph nodes, mural fat, and creeping fat were considered relevant in diagnosing proctitis on pelvic MRI, as they showed a significant correlation between at least two observers and the endoscopy reference standard, and at least a good interobserver agreement for at least two of 


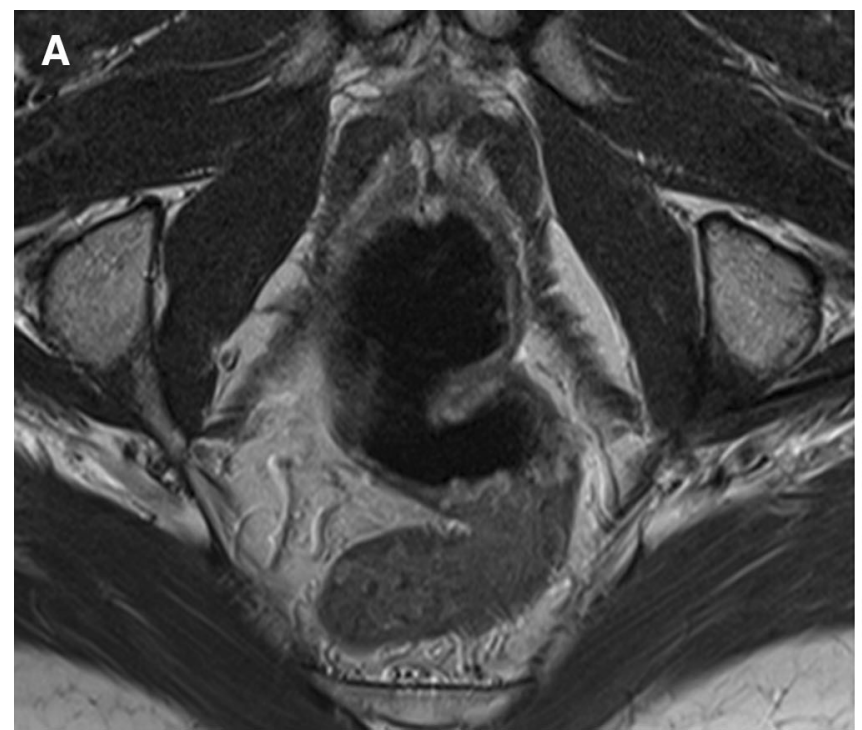

Fig. 6. A 45-year-old male with Crohn's disease and no signs of proctitis at endoscopy. A Axial oblique T2-weighted image and $\mathbf{B}$ axial oblique fat-saturated T2-weighted image

three observer pairs. Perimural T2 signal and perimural enhancement showed a significant correlation for all the three observers and a moderate interobserver agreement for at least two of the three observer pairs. Mural T2 signal and $\mathrm{T} 1$ enhancement degree and pattern showed poor to moderate reproducibility.

This is to our knowledge, the first study reporting on the specific MRI features associated with proctitis on a dedicated pelvic MRI. Previous research did study rectal involvement in Crohn's disease patients, but this was done using MR enterography or MR colonography [8-10]. No specific rectal and/or perirectal features were described. Van Assche developed an MRI-based score of perianal Crohn's disease severity with rectal wall thickening as the sole indicator for rectal inflammation, which can be used for evaluation of response to treatment $[16,17]$. Our results confirmed the correlation between rectal wall thickening and inflammation; in addition, a moderate to good interobserver agreement was observed. Most features considered relevant in diagnosing proctitis involved the mesorectal fat tissue (Figs. 4, 5). This is in contrast to a study that showed only fair reproducibility for perimural features on MR enterography [11]. Crohn's disease is known for its transmural inflammation and subsequent perimural involvement. In the rectum, this perimural involvement was often quite prominent. This might be related to the isolated localization of the rectum surrounded by mesorectal fat tissue, where perimural changes are somewhat easier appreciated than when multiple loops of bowel are closely aligned. Further, the rectum was not or moderately distended in most cases, which might result in the perirectal features becoming more apparent. This rectal collapse might have led to increased wall thickness measurements. Even so, there was a significant difference

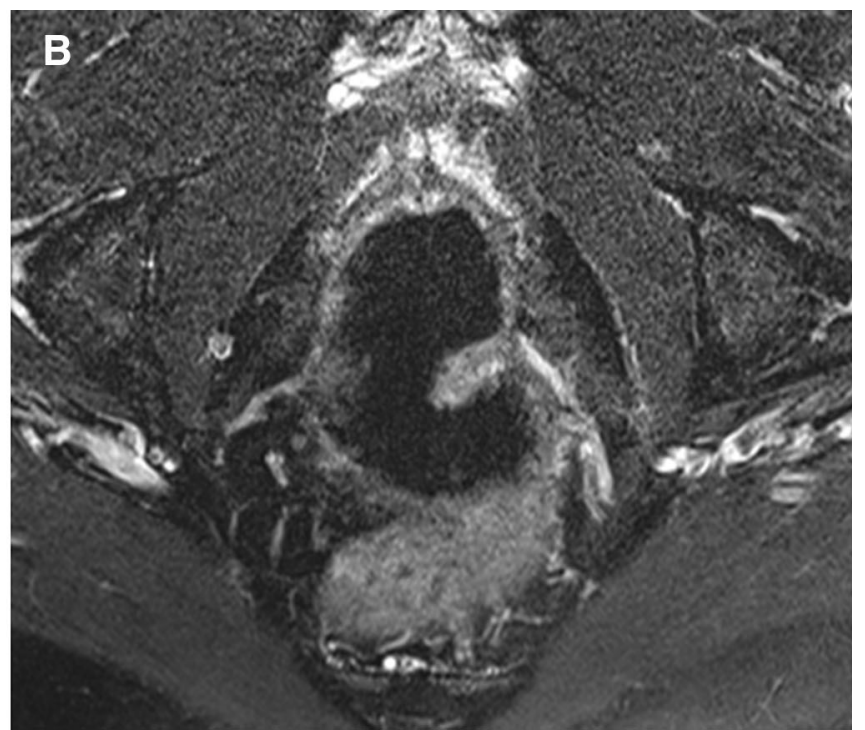

obtained at the same level shows a moderate increase of T2 signal intensity of the rectal wall.

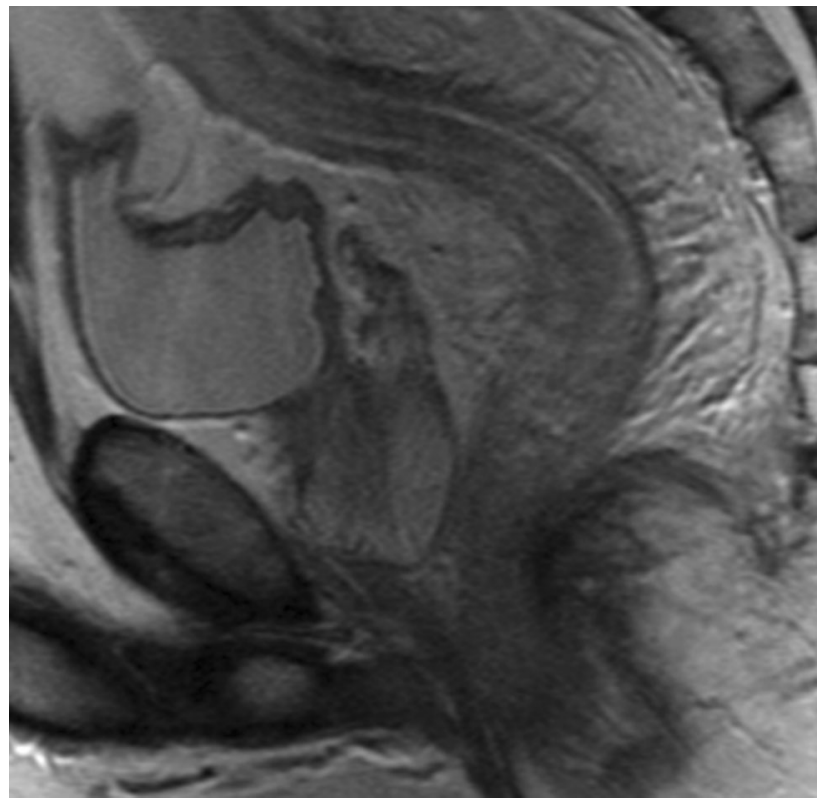

Fig. 7. A 43-year-old male with Crohn's disease and ulcerative proctitis at endoscopy. Sagittal T2-weighted image shows the increased perimural vascularity perpendicular to the rectum ('comb sign') in addition to the wall thickening of the entire rectum.

in wall thickness in proctitis versus no proctitis as observed by all three observers. Further research should focus on the predictive value of the individual MRI features identified in our study, and the clinical use in monitoring treatment response as a non-invasive alternative to endoscopy and in case of severe anal stenosis.

In contrast, luminal features already proven to be useful in establishing disease severity on MR enterography 
and MR colonography, for example, T2 signal intensity and $\mathrm{T} 1$ enhancement (pattern and degree), were considered not useful in our study [7-9]. In order to decide if a certain feature is considered normal or increased, one must be able to compare it to other colonic loops, which were almost never included in the field of view of the T1weighted and T2-weighted fat-saturated sequences that was only performed in the axial oblique plane. Also, in our standard perianal fistula protocol, no T1-weighted precontrast images for comparison were performed. For the T2 signal intensity of the rectal wall in almost all cases (normal or proctitis), observers scored the $\mathrm{T} 2$ signal intensity of the rectal wall at least as slight, but mostly as moderately increased (Fig. 6). This suggests that the normal rectal signal intensity is already light gray on T2 fatsaturated images and that the grading scale used for luminal disease was not adequate for the rectum. Furthermore, MR enterography and colonography use luminal contrast to obtain bowel distention.

We do not have a balanced explanation for the lack of correlation between the supralevatoric extension of fistula and/or abscess and the presence of proctitis as one might have expected. Only our most experienced observer showed a significant correlation for the presence of ulcerations. A limitation for this feature is that in the proctitis group, we combined the patients with nonulcerative and ulcerative proctitis because of low number of patients in each group. As the number of ulcerative proctitis cases was low, a possible correlation in ulcerative proctitis might not be identified. Combining ulcerative and non-ulcerative proctitis, also prevented us to rule on disease severity. For the features 'presence of creeping fat' and 'comb sign,' we had no circumscribed definitions or grading, and observers had to score these features according to their expert opinion rendering it susceptible for subjectivity. Although the comb sign did show a significant correlation with endoscopy, the interobserver agreement was very low for two of three observer pairs, probably because of unfamiliarity with this feature (Fig. 7).

Increased wall thickness is not only seen in the active phase of Crohn's disease but also in the chronic stage of the disease. Also, the presence of mural fat and creeping fat are signs of chronic disease. The significant correlation to active inflammation at endoscopy for these features is inherent to Crohn's disease with a chronic course of relapsing and remitting inflammation, where features of chronic disease coexist with acute inflammatory changes.

Our study has several limitations. First, endoscopy reports were retrospectively analyzed in order to determine the reference standard. However, only reports with evident mentioning of the rectum were included and evaluated using a predefined clear-cut scoring system [10] and an experienced gastroenterologist was involved. Because of only including patients with mentioning of the rectum in the endoscopy report, our patient popu- lation was subject to selection bias creating a diseaseenriched population. Second, the time between the MRI and the endoscopy examination ranged from 0 to 44 days. In this time frame disease activity could have been altered because of natural course. However, since we excluded all patients with change in medical or surgical therapy during the time interval, this possibility was minimized. Third, the use of a kappa/intraclass coefficient value of $\geq 0.60$ would have strengthened our study, but at initial evaluation of our data too little features remained. In this first phase of identifying possible relevant features, we wanted to include as much features as possible, in order to make a further selection in a future validation study. Fourth, the introductory session for the observers regarding the different MRI features was held by the same expert abdominal radiologist (JS who was also one of the readers in this study), which could have increased reproducibility. We did not notice higher agreement between the observer pairs including this expert abdominal radiologist than the other observer pair.

In conclusion, for diagnosing proctitis in Crohn's disease in perianal MRI, MRI features involving the mesorectal tissue, perimural T2 signal, perimural T1 enhancement, the presence of creeping fat, and size of mesorectal lymph nodes were most valuable, as they showed a significant correlation with endoscopical findings and were reproducible. Established luminal features like mural T2 signal and T1 enhancement may be less helpful in perianal MRI. In addition, rectal wall thickness and presence of mural fat were relevant as well. Future research should focus on these MRI features by validating them in a prospective study and for defining thresholds for continuous variables.

Acknowledgments. We would like to thank research nurse Jitteke de Jong for her help in performing the search in the hospitals' patient database and physician A. J. Nederveen for his help in extracting the MRI scan parameters from the DICOM headers.

\section{Compliance with ethical standards}

Funding This study received no funding. For none of the authors of this manuscript any financial interest is applicable.

Disclosures Professor J. Stoker is a consultant for Robarts. Dr.C.Y. Ponsioen has received unconditional grants from Takeda, served as a consultant for Takeda and Abbvie, and received speaker's fees from Takeda, MSD, Ferring, Dr. Falk, and Abbvie. All other authors, C.J. Tutein Nolthenius, S. Bipat, B. Mearadji, S. Spijkerboer, and A.D. Montauban van Swijndregt, have nothing to disclose.

Informed consent The requirement for review by the Medical Ethical Committee or informed consent was waived because of the retrospective nature of this study with pre-existing data.

Open Access This article is distributed under the terms of the Creative Commons Attribution 4.0 International License (http://creative commons.org/licenses/by/4.0/), which permits unrestricted use, distribution, and reproduction in any medium, provided you give appropriate credit to the original author(s) and the source, provide a link to the Creative Commons license, and indicate if changes were made. 


\section{Appendices}

Appendix A. Range of MR scan parameters performed on three different MR scanners

\begin{tabular}{|c|c|c|c|c|c|}
\hline & $\begin{array}{c}\text { Sagittal } \\
\text { T2-weighted } \\
\text { TSE }\end{array}$ & $\begin{array}{c}\text { Coronal } \\
\text { T2-weighted } \\
\text { TSE }\end{array}$ & $\begin{array}{c}\text { Axial } \\
\text { T2-weighted } \\
\text { TSE }\end{array}$ & $\begin{array}{c}\text { Axial } \\
\text { T2-weighted } \\
\text { TSE with fat sat. }\end{array}$ & $\begin{array}{l}\text { Axial T1-weighted } \\
\text { TSE with fat sat. }+ \\
\text { iv contrast } \\
\text { enhancement }^{\mathrm{a}}\end{array}$ \\
\hline \multicolumn{6}{|c|}{$1.5 \mathrm{~T}$ (Siemens, Avanto), $33 \mathrm{MR}$ scans } \\
\hline Field of view $(\mathrm{cm})$ & 300 & $300 / 320$ & $220 / 300$ & $220 / 300$ & $300-450$ \\
\hline No. of slices & $28-40$ & $25-42$ & $28-45$ & $28-42$ & $28-42$ \\
\hline Repetition time (ms) & $2500-4000$ & $2500-4000$ & $2500-4000$ & $2500-4000$ & $600-718$ \\
\hline Echo time (ms) & $69 / 70$ & $70-121$ & $70-121$ & $70-121$ & $9,4 / 11$ \\
\hline Image matrix & $512 \times 231-512 \times 578$ & $256 \times 297-512 \times 575$ & $256 \times 297-512 \times 578$ & $256 \times 297-512 \times 575$ & $256 \times 288-512 \times 256$ \\
\hline Slice thickness (mm) & $3 / 4$ & $3 / 4$ & $3 / 4$ & 4 & 4 \\
\hline Slice gap $(\mathrm{mm})$ & 0.4 & $0-0,4$ & $0-0.4$ & $0 / 0.4$ & $0-0,4$ \\
\hline NSA & $1-3$ & $1 / 2$ & $1 / 2$ & $1 / 2$ & $1-4$ \\
\hline Flip angle & $90 / 150$ & $90 / 150$ & $90 / 150$ & $90 / 150$ & $90 / 150$ \\
\hline \multicolumn{6}{|c|}{$1.5 \mathrm{~T}$ (Signa, GE), $20 \mathrm{MR}$ scans } \\
\hline Field of view $(\mathrm{cm})$ & 300 & 300 & 300 & 300 & $300 / 450$ \\
\hline No. of slices & 32 & 32 & $31 / 32$ & $28-32$ & $31 / 32$ \\
\hline Repetition time (ms) & 2500 & 2500 & 2500 & $2500-4500$ & $560 / 600$ \\
\hline Echo time (ms) & $69 / 70$ & $71 / 72$ & 69 & $69-83$ & 10.8 \\
\hline Image matrix & $512 \times 200$ & $512 \times 200$ & $512 \times 200$ & $256 \times 100$ & $256 \times 100$ \\
\hline Slice thickness (mm) & 4 & 4 & 4 & 4 & 4 \\
\hline Slice gap $(\mathrm{mm})$ & 0.4 & 0.4 & 0.4 & 0.4 & 0.4 \\
\hline NSA & 1 & 1 & 1 & 1 & 2 \\
\hline Flip angle & 90 & 90 & 90 & 90 & 90 \\
\hline \multicolumn{6}{|l|}{$3 \mathrm{~T}$ (Philips), $5 \mathrm{MR}$ scans } \\
\hline Field of view $(\mathrm{cm})$ & $230-360$ & $240-400$ & $240-400$ & $240-400$ & $300 / 400$ \\
\hline No. of slices & $30-41$ & $30-37$ & $32-45$ & $35-45$ & $40-45$ \\
\hline Repetition time (ms) & $2689 / 3000$ & $2689-3000$ & $2689-3000$ & $3000 / 4626$ & $550-786$ \\
\hline Echo time (ms) & $70-100$ & $70-100$ & $70-100$ & $70 / 100$ & 10 \\
\hline Image matrix & $256 \times 198-528 \times 361$ & $400 \times 246-528 \times 400$ & $400 \times 395-528 \times 361$ & $300 \times 287-768 \times 287$ & $248 \times 248-512 \times 255$ \\
\hline Slice thickness (mm) & $3 / 4$ & $3 / 4$ & $3 / 4$ & $3 / 4$ & $3 / 4$ \\
\hline Slice gap $(\mathrm{mm})$ & $0-1$ & $0 / 0.3$ & $0 / 0.3$ & $0 / 0.3$ & $0 / 0.3$ \\
\hline NSA & $2 / 4$ & $1 / 4$ & $1 / 4$ & $1 / 2$ & $1 / 2$ \\
\hline Flip angle & 90 & 90 & 30 & 90 & 90 \\
\hline
\end{tabular}

${ }^{a}$ With the exception of one SENSE dixon post-contrast series: FOV 400, no. of slices 480, TR 0, TE 0, matrix 512x207, slice thickness 1.5 , gap 0, averages 1 , flip angle 8

Appendix B. Combined results of interobserver agreement and correlation between observers and reference standard

\begin{tabular}{|c|c|c|c|c|c|c|c|}
\hline \multirow[t]{2}{*}{ MRI features } & \multicolumn{3}{|c|}{$\begin{array}{l}\text { Significant correlation between observer and } \\
\text { reference standard }(p \leq 0.05)\end{array}$} & \multicolumn{3}{|c|}{$\begin{array}{c}\text { Kappa or intraclass coefficient } \\
\geq 0.40\end{array}$} & \multirow[t]{2}{*}{ Relevant MRI feature } \\
\hline & Observer 1 & Observer 2 & Observer 3 & 1 vs. 2 & 2 vs. 3 & 1 vs. 3 & \\
\hline Wall thickness & Yes & Yes & Yes & Yes & Yes & Yes & Yes \\
\hline Mesorectal lymph nodes & Yes & Yes & Yes & Yes & Yes & Yes & Yes \\
\hline Obturator lymph nodes & No & No & No & No & No & No & No \\
\hline Iliac lymph nodes & No & No & No & No & No & No & No \\
\hline Inguinal lymph nodes & No & No & No & Yes & No & Yes & No \\
\hline$\%$ of circumference involved & Yes & Yes & Yes & No & No & Yes & No \\
\hline Mural T2 signal & Yes & Yes & No & No & $\mathrm{No}^{\mathrm{a}}$ & $\mathrm{No}^{\mathrm{a}}$ & No \\
\hline Perimural T2 signal & Yes & Yes & Yes & Yes & Yes & Yes & Yes \\
\hline T1 enhancement & Yes & No & Yes & No & No & No & No \\
\hline T1 enhancement pattern & No & Yes & Yes & No & No & Yes & No \\
\hline Perimural enhancement & Yes & Yes & Yes & Yes & No & Yes & Yes \\
\hline Mural fat & Yes & No & Yes & Yes & Yes & Yes & Yes \\
\hline Ulcers & No & No & Yes & $\mathrm{No}^{\mathrm{a}}$ & No & No & No \\
\hline Supralevatoric fistula & No & No & No & Yes & Yes & Yes & No \\
\hline Supralevatoric abscess & No & No & No & Yes & Yes & Yes & No \\
\hline Creeping fat & b & Yes & Yes & Yes & Yes & Yes & Yes \\
\hline Comb sign & Yes & Yes & Yes & No & No & Yes & No \\
\hline
\end{tabular}

${ }^{a}$ Proportion of agreement calculated, because observed concordance is smaller than mean-chance concordance. Assuming kappa $\leq 0.40$

${ }^{b}$ Not calculated because of the presence of a zero in the crosstab 


\section{References}

1. Beets-Tan RG, Beets GL, van der Hoop AG, et al. (2001) Preoperative MR imaging of anal fistulas: does it really help the surgeon? Radiology 218(1):75-84

2. Panes J, Bouhnik Y, Reinisch W, et al. (2013) Imaging techniques for assessment of inflammatory bowel disease: joint ECCO and ESGAR evidence-based consensus guidelines. J Crohn's Colitis 7(7):556-585

3. Gecse K, Khanna R, Stoker J, et al. (2013) Fistulizing Crohn's disease: diagnosis and management. United Eur Gastroenterol J 1(3):206-213

4. Buchanan GN, Halligan S, Bartram CI, et al. (2004) Clinical examination, endosonography, and MR imaging in preoperative assessment of fistula in ano: comparison with outcome-based reference standard. Radiology 233(3):674-681

5. Schwartz DA, Herdman CR (2004) Review article: the medical treatment of Crohn's perianal fistulas. Aliment Pharmacol Ther 19(9):953-967

6. Safar B, Sands D (2007) Perianal Crohn's disease. Clin Colon Rectal Surg 20(4):282-293

7. Steward MJ, Punwani S, Proctor I, et al. (2012) Non-perforating small bowel Crohn's disease assessed by MRI enterography: derivation and histopathological validation of an MR-based activity index. Eur J Radiol 81(9):2080-2088

8. Ajaj WM, Lauenstein TC, Pelster G, et al. (2005) Magnetic resonance colonography for the detection of inflammatory diseases of the large bowel: quantifying the inflammatory activity. Gut 54(2):257-263
9. Rimola J, Rodriguez S, Garcia-Bosch O, et al. (2009) Magnetic resonance for assessment of disease activity and severity in ileocolonic Crohn's disease. Gut 58(8):1113-1120

10. Rimola J, Ordas I, Rodriguez S, et al. (2011) Magnetic resonance imaging for evaluation of Crohn's disease: validation of parameters of severity and quantitative index of activity. Inflamm Bowel Dis 17(8): 1759-1768

11. Tielbeek JA, Makanyanga JC, Bipat S, et al. (2013) Grading Crohn disease activity with MRI: interobserver variability of MRI features, MRI scoring of severity, and correlation with Crohn disease endoscopic index of severity. AJR Am J Roentgenol 201(6):1220-1228

12. Tolan DJ, Greenhalgh R, Zealley IA, Halligan S, Taylor SA (2010) MR enterographic manifestations of small bowel Crohn disease. Radiographics 30(2):367-384

13. Szurowska E, Wypych J, Izycka-Swieszewska E (2007) Perianal fistulas in Crohn's disease: MRI diagnosis and surgical planning: MRI in fistulazing perianal Crohn's disease. Abdom Imaging 32(6):705-718

14. Horsthuis K, Stoker J (2004) MRI of perianal Crohn's disease. AJR Am J Roentgenol 183(5):1309-1315

15. Altman DG (1999) Practical statistics for medical research. Boca Raton: Chapman \& Hall

16. Van Assche G, Vanbeckevoort D, Bielen D, et al. (2003) Magnetic resonance imaging of the effects of infliximab on perianal fistulizing Crohn's disease. Am J Gastroenterol 98(2):332-339

17. Horsthuis K, Ziech ML, Bipat S, et al. (2011) Evaluation of an MRI-based score of disease activity in perianal fistulizing Crohn's disease. Clin Imaging 35(5):360-365 